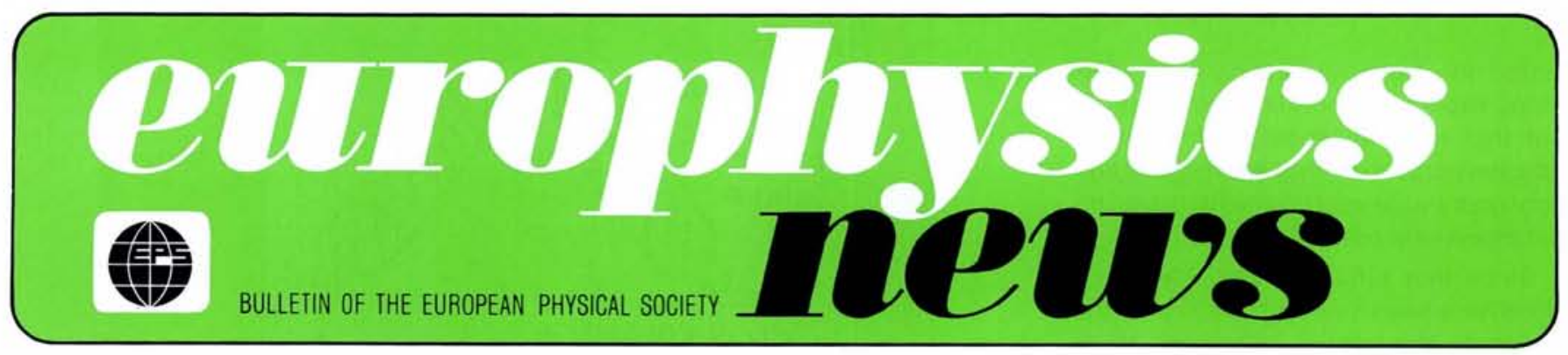

J.A.

\title{
Experiments of Parity Non-Conservation in Heavy Atoms
}

\author{
D.N. Stacey, Oxford
}

(Clarendon Laboratory)

According to the text-books, atomic states have well-defined parity, and certainly to many people the idea that atoms do not possess reflection symmetry is counter-intuitive. What can the electrons be doing which allows one to distinguish between an atom and its mirror image? (A partial answer to this question is illustrated in Fig. 1.) Whatever the conceptual difficulties involved, the idea that parity non-conserving (PNC) effects may occur in atomic phenomena has in fact been discussed for many years; indeed, one of the methods used to study such effects in modern experiments, optical rotation, was suggested by Zel-dovitch as long ago as 1959 . However, the current activity in this field was prompted by the success of unified gauge theories of the weak and electromagnetic interactions. Although this theoretical approach was proposed by Glashow, Weinberg and Salam in the late sixties, it was some years before relevant atomic physics experiments got under way, stimulated by two developments. First, processes mediated by the weak neutral current, predicted by the Weinberg-Salam theory, were found in neutrino scattering experiments. $(\mathrm{Ob}$ servable atomic PNC effects depend on the existence of a neutral component of the weak interaciton.) Second, M.A. and C. Bouchiat in Paris showed that there was a $Z^{3}$ scaling of certain PNC effects, leading to phenomena which should be detectable in heavy atoms with modern techniques based on tunable lasers.

In the decade which has passed since then, several experiments designed to study PNC effects have been carried out. By any reasonable standards, progress has been remarkable. The effects are very small - much smaller than they were at first predicted to be - but nevertheless they have been shown to exist, and the quoted accuracy of the most recent measurements is better than $20 \%$. Despite this, the work got off to an unfortunate start in its relationship to elementary particle theory. The initial results were eagerly awaited, since they were expected to provide the first evidence that the weak neutral current was parity violating. In the event, the earliest data (from optical rotation experiments in bismuth at Oxford and Seattle) appeared to do just the opposite, leading to considerable surprise and some confusion. Elementary particle theorists, at first taken aback, soon produced a spate of papers presenting ingenious models of the electro-weak interaction which were consistent with a null result in bismuth. This ingenuity, alas, has since been proved unnecessary.

It is of course easy to blame the experimentalists for this misleading evidence. The measurements are difficult and the first results were unreliable because of imperfectly understood systematic effects. However, at least as important a contributing factor was the unexpected smallness of the optical rotation. The earliest theoretical predictions overestimated the effect considerably, partly because of the difficulties involved in the atomic calculations, partly

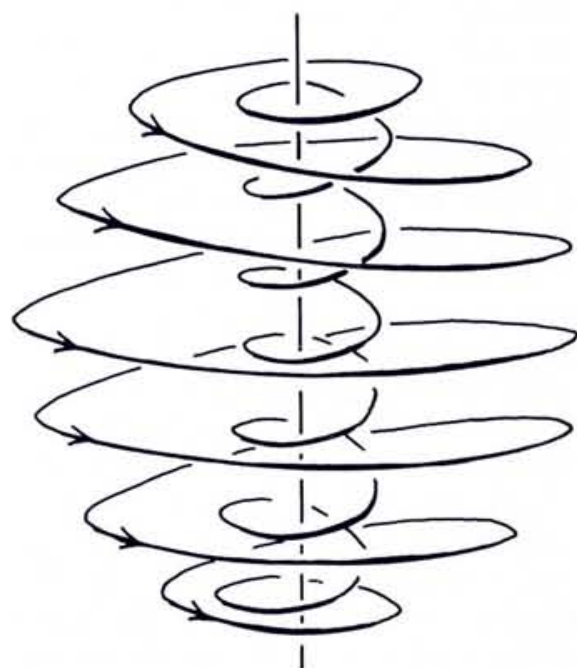

Fig. 1 - Parity non-conservation in the hydrogen atom. The sketch shows a flow line of the probability current for an electron in a $2 p$ state of atomic hydrogen, taking account of the admixture of $2 s$ caused by $Z^{\circ}$ interchange between the electron and the proton (the admixture has been scaled up by a factor of about $10^{10}$ for the purposes of the diagram). The motion has a definite handedness, making the real atom fundamentally different from its mirror image which does not occur in nature.

because of the poor accuracy with which the so-called Weinberg angle was known. (This angle is a parameter of the theory which had at that time been eva-

\section{Contents}

Experiments on Parity NonConservation in Heavy Atoms 1

Europhysics Letters

Editor-in-Chief

Theory of Resonances in Molecular Systems

Big Detectors of BGO

Muons as Probes in Solids

EPS Conferences in 1985

Loss of EPS Worker

ECA Series 9 
luated only from the early neutrino scattering experiments.) It is worth pointing out that even the most recent optical rotation measurements are more nearly consistent with zero than with those initial theoretical predictions.

Since that time, non-zero PNC rotations have been reported, the first by the group at Novosibirsk. However, there has been a history of disagreement between the results from different laboratories and not surprisingly, many elementary particle physicists became wary of the atomic physics experiments. They were much relieved when the work on deep inelastic scattering of polarized electrons by deuterium at SLAC showed a convincing PNC asymmetry in agreement with the Weinberg-Salam theory. Subsequently, the theory has had further successes, culminating in the direct observation of the $W^{ \pm}$and $Z^{0}$, the particles which together with the photon mediate the electro-weak interaction. Despite this, the level of activity on the atomic experiments has if anything, intensified, and it is interesting to consider why this should be so. Have atomic PNC effects now any relevance to elementary particle physics? Have they even any relevance to atoms? To answer these questions, we have to look at the experiments and the theory in more detail.

PNC phenomena in atoms come about because of the $\mathrm{Z}^{0}$-exchange between electrons and nucleons (there is also an electron-electron interaction, but it generally gives rise to much smaller effects). There are four coupling constants $C_{1 n}, C_{2 n}, C_{1 p}$ and $C_{2 p}$ (two each for the neutron and proton). The interactions characterized by $C_{1 \mathrm{n}}$ and $C_{1 \mathrm{p}}$ obey the $Z^{3}$ law; they do not depend on the nucleon spin and so are scalarly additive for the nucleons in a heavy nucleus. The other interactions have only a $Z^{2}$ scaling because the resultant nuclear spin is only of the order of the value characteristic of a single nucleon. When one takes account also of the fact that according to the Weinberg-Salam theory, $C_{1 n}$ is much larger than the other coupling constants, it is not surprising that all heavy atom experiments have so far been designed to measure effects primarily due to the interaction characterized by $C_{10}$.

This interaction has a small but profound influence on the electronic structure of the atom. An electronic state which would be a parity eigenstate in the absence of the neutral current, acquires a component of opposite parity. This gives rise to the kind of behaviour shown in Fig. 1. For light atoms the magnitudes of the admixture coeffi-

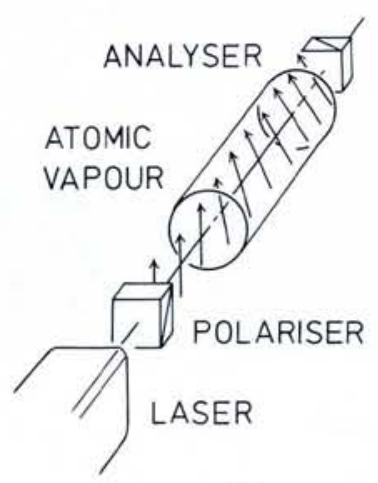

(a)

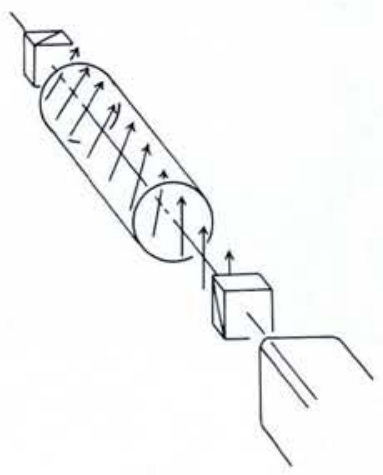

(b)

Fig. 2 - The scheme of an optical rotation experiment. One looks for a rotation of the plane of polarized light as it passes through an atomic vapour. To see the parity violating nature of the phenomenon, consider the two experiments (a) and (b) which differ only in that they are mirror images of each other and hence show rotation in opposite senses. If the vapour were molecular, both of these experiments would be realizable in practice because an optically active molecule always exists in two forms which are mirror images of each other. However, this is not the case for atoms (see Fig. 1) so if (a) represents what actually occurs under given conditions, it is impossible to set up the system (b) which exhibits a rotation in the opposite sense.

cients are of order $\left(G_{F} a_{0}{ }^{-3}\right) \alpha / \triangle E\left(G_{F}\right.$ and $\alpha$ are the weak interaction and the electromagnetic fine structure constants, respectively, $a_{0}$ the Bohr radius and $\triangle E$ the energy separation of the mixed parity eigenstates). This gives a typical value of $10^{-15}$ way beyond the reach of any experiment. This is why the early contribution of the Bouchiats was of crucial importance; in heavy elements the $Z^{3}$ scaling leads to values of around $10^{-10}$. One then finds that it is possible to devise experiments with the necessary sensitivity, the methods adopted giving rise to a further enhancement. The general scheme is to probe a weak transition which, because of the PNC admixture, exhibits a difference in response to left and right circularly polarized light. This comes about through interference; there are two amplitudes for the transition, the regular amplitude and that due to the PNC admixture. The enhancement occurs because in the cases chosen for study, the smallness of the PNC admixture is partially offset by the greater intrinsic strength of the admixed component of the transition.

There have been two types of experiment carried out so far which we may call fluorescence and optical rotation. In both, atoms are subjected to polarized light in the vicinity of the weak transition. However, in the case of optical rotation, one observes the effect of the atoms on the radiation while for fluorescence the opposite is true. We begin by discussing the fluorescence approach; the results so far reported have been for caesium $6 s^{2} S_{1 / 2}-7 s^{2} S_{1 / 2}$ (Paris) and thallium $6 p^{2} P_{1 / 2}-7 p^{2} P_{1 / 2}$ (Berkeley).

The experiments have gone through several phases and are still developing. Conceptually the simplest scheme is to shine light through a vapour cell and observe the difference in fluorescent intensity as the sense of the circular polarization is reversed. This arrangement can give a very large fractional effect about $10^{-4}$ for caesium - but, in fact, the experiment has not been carried out in quite this form because the transitions of interest are too weak. The solution was to add an electric field, which increases the transition rate in a controlled fashion (at the expense of reducing the fractional PNC effect). It also changes the PNC signature of the fluorescence from modulation of intensity to modulation of polarization. The modulation is observed directly in the caesium experiment, but in thallium it is advantageous to excite the atoms to a still higher level by means of a second laser and monitor the subsequent fluorescence. In the most recent work on thallium the atoms are subjected to crossed $E$ and $B$ fields, and the atoms illuminated with modulated linearly polarized light. This approach has a number of advantages, including a simpler detection scheme. The uncertainty quoted with the most recent measurements is rather better than $20 \%$ for both elements, the results being in agreement with the predictions of the Weinberg-Salam theory.

We now turn to optical rotation. The difference in response to left and right circularly polarized light gives rise to a rotation of the plane of polarization of radiation traversing an atomic vapour. Fig. 2 shows why the effect is parity violating. The rotation under typical conditions rises to about $10^{-7}$ radians in the vicinity of the transitions studied. The PNC rotation (near a single isolated spectral component) has a dispersive shape, and all measurements of the ef- 
fect exploit its variation with frequency to discriminate against rotations originating in other ways.

There are detailed variations in the experimental methods adopted, but the principle is always the same; the atomic vapour is contained in an oven between nearly crossed polarizers. The light source is a tunable laser. The optical rotation is found from the intensity transmitted through the system which depends on the laser frequency and on the number of atoms in the line of sight. The latter is found from the Faraday effect, the rotation induced by an applied axial $B$-field.

The method is very sensitive but it is difficult to discriminate against spurious effects. It is amusing to reflect that a measurement accurate to about 10\% could be carried out in less than a minute if one were limited only by noise in the signal. In fact, some of the optical rotation experiments have been going for well over $10^{6}$ minutes and this accuracy has still not been reached. So far, measurements have been made at 648 $\mathrm{nm}$ and $876 \mathrm{~nm}$ in bismuth and $1.28 \mu \mathrm{m}$ in lead; all groups involved have found optical rotations of the order of magnitude predicted, the quoted experimental uncertainty ranging from $15 \%$ to $30 \%$. The bismuth $648 \mathrm{~nm}$ transition is of particular interest since it is the only atomic PNC experiment for which results have been reported from different laboratories, at Oxford, Moscow and Novosibirsk. Until quite recently, all three disagreed completely. The latest data from Moscow and Oxford are consistent while the Novosibirsk group still quotes an effect of about a factor of two larger. Whatever the final outcome, this shows the importance of cross-checks between different laboratories when such small effects are being measured and the possibility of significant systematic error is so difficult to exclude. A further useful comparison will be possible when the Oxford group obtain a result for the $876 \mathrm{~nm}$ transition in bismuth, already studied in Seattle. Checks of the fluorescence experiments are provided by the consistent results obtained when techniques have been varied and when different hyperfine transitions have been studied. However, even here confirmation of the results from other laboratories would be valuable.

At the same time, it does now seem likely that the major problems in the various experiments have been recognised, and that before long the atomic physics data will be accurate to $10 \%$ or better. We therefore turn again to the question of the motivation for all this effort.

From the elementary particle point of view, the interest is in the measurement of the coupling constants. As mentioned before, the heavy atom results depend mainly on one component of the neutron-electron interaction, but one can write down the exact combination of quark-lepton coupling constants which determines the PNC effect in any given case. The first point to make is that the combinations measured in the heavy atom experiments are almost orthogonal to that obtained in the electron scattering work at SLAC. The various methods of studying the electro-weak interaction are complementary, and taken together can in principle distinguish between different gauge models.

How far "in principle" can become "in practice" depends critically on the accuracy of the atomic experiments. For example, if the combined uncertainties in the experiments and atomic theory can be brought below $10 \%$, one can derive a value of the $Z^{0}$ mass with a precision comparable with that of the existing CERN result. If it is possible to reach the level of a few percent, the experiments even become sensitive to radiative corrections to the theory. Furthermore, one may eventually hope to observe nucleon spin-dependent effects and even perhaps the electron-electron interaction. However, there is one major difficulty to be overcome, which is to solve the atomic problem well enough to derive the coupling constants from the measured PNC effects to a precision comparable with the expected experimental accuracy. Even for the comparatively simple systems of caesium and thallium, the accuracy of the calculations is hard to assess, though errors below $15 \%$ have been quoted. This theoretical work, is now being pursued vigorously, one object being to compare the results of several different approaches to the same problem. This should give some feeling for the reliability of the calculations and how best to go about them. One may look for significant improvements over the next few years so the prospect of a critical comparison between high and low energy data looks promising.

Nevertheless, it is important to stress that the interest of the field is more general than this. Naturally, atomic physicists hope that their measurements of PNC effects will contribute to our understanding of the basic physical laws; it is reassuring to find that the atom still maintains its role as a natural testing round for new theoretical concepts. The theories developed to ac- count for high energy phenomena often seem to have little relevance outside the domain of giant accelerators, and it is striking that the familiar atom, which is often said these days to be completely understood in principle, should be so profoundly affected by the existence of the weak neutral current. However, quite apart from these fundamental considerations there have already been major benefits from this field of study. New experimental techniques have been developed which have applications in quite different fields. Also, as already remarked, the need for theoretical progress has stimulated considerable activity which will give insights into the description of many other atomic phenomena. There is something of a temptation to assume that PNC effects are only interesting in so far as they might influence elementary particle theory. This is nonsense; the fascination of the great majority of atomic phenomena depends not at all on their capacity to change our views on fundamental interactions. The idea of atoms without reflection symmetry is a strange and beautiful one in its own right, and must surely rank as one of the most striking developments in the distinguished tradition of atomic physics.

\section{Further Reading}

The field has been reviewed by Fortson E.N. and Wilets L. in Advances in Molecular Physics 16 (1980) 319 .

A shorter account containing references to more recent work is given by the present author in Acta Physica Polonica A66 (1984) 4, 377.

Bouchiat M.A. and Pottier L. have given an account for non-specialists in Scientific American 250 (June 1984) 6, 76.

\section{Europhysics Letters}

\section{Editor-in-Chief}

Following discussions throughout the European Physical Society - the Divisions, the national societies, members of Council and the partners investing in the journal - Nicholas Kurti of Oxford has been invited by the Executive Committee to become the first Editor-inChief of the EPS general physics letters journal to be launched in January 1986.

$\mathrm{He}$ has accepted and with characteristic energy and enthusiasm begun to grapple with the questions raised by the implementation of the structure that has been agreed. Readers will recall that this comprises a body of Co-editors each directly responsible for accepting or rejecting submitted letters in a given subject area, supported by a group of Advisory Editors with wide geographical and subject background. 